\title{
TRANSFERT EXPÉRIMENTAL D'HELMINTHES ADULTES CHEZ LES POISSONS PAR ICHTYOPHAGIE ET CANNIBALISME
}

\author{
I. DE BURON*, C. MAILIARD*
}

\begin{abstract}
RÉSUMÉ. Le passage expérimental d'Acanthocéphales et de Trématodes adultes, parasites intestinaux de Poissons, peut s'effectuer dans un Poisson prédateur sans altérations pour ces Helminthes. Cette expérience appliquée à des P’oissons d'espèces différentes (cannibalisme ou ichtyophagie) montre qu'elle peut être utilisée comme nouvelle technique d'approche de la spéciation de ces parasites et de leur spécificité pour leurs hôtes définitifs.
\end{abstract}

Experimental transfer by ichtyophagy and cannibalism of adult helminths, parasite of fishes.

SUMMARY. Adult intestinal parasites (acanthocephalans and digeneans) of fishes can be experimentaly transfered to predator fishes without any alteration. This experiment carried out with different species of fishes (ichtyophagy or cannibalism) can be used as a new technic of approach to the speciation of these helminths and their specificity for their definitive hosts.

Chez les Helminthes, et en particulier chez les Trématodes et les Acanthocéphales, les critères taxinomiques classiques, essentiellement morpho-anatomiques, ne permettent pas toujours la distinction indiscutable des espèces entre elles. Il en résulte une systématique souvent confuse, sans cesse remodelée au fur et à mesure que s'affinent les descriptions des différents parasites.

Les techniques génétiques modernes permettent de séparer avec certitude les espèces occultes (Ayala, 1975, Pasteur et Pasteur, 1980). Ainsi, en ce qui concerne les Helminthes, l'étude électrophorétique de la population d'Acanthocéphales parasites d'Arnoglossus laterna (Bothidés) a permis de distinguer une espèce nouvelle, Acanthocephaloides geneticus, jusqu'alors confondue avec $A$. propinquus récoltée chez Gobius niger (Buron, Renaud et Euzet, 1985). Ces techniques s'avèrent

* Laboratoire de Parasitologie comparée, Université des Sciences et Techniques du Languedoc, place E.-Bataillon, F 34060 Montpellier Cedex.

Accepté le 14 octobre 1986. 
donc très efficaces quant à l'approche du phénomène de spéciation mais nécessitent des manipulations délicates ne s'appliquant pas à tous les matériels biologiques.

La connaissance imparfaite de la systématique accentuée par l'ignorance de la majorité des cycles évolutifs des helminthes de poissons entraîne une méconnaissance de la spécificité des parasites vis-à-vis de leurs hôtes définitifs. Cette spécificité, notion importante en parasitologie, a fait l'objet de 2 symposiums (Neuchàtel, 1957², Paris, 1981²). La spécificité des Helminthes parasites peut être considérée comme étant la conséquence de la reconnaissance d'un milieu favorable à la réalisation de leur niche écologique, donc d'un milieu sélectif permettant la maturité sexuelle et la reproduction.

Chez les Poissons, les phénomènes d'ichtyophagie intraspécifique (cannibalisme) ou interspécifique nous ont permis de mettre en évidence expérimentalement l'intérêt de la spécificité parasitaire en tant que critère taxinomique.

\section{Matériel et méthodes}

Les expériences que nous avons menées ont été pratiquées sur des Trématodes du genre Helicometra Odhner, 1902 et des Acanthocéphales du genre Acanthocephaloides Meyer, 1932 présents chez différentes espèces de Poissons dans le bassin de Thau, lagune méditerranéenne du sud de la France.

Dans le genre Helicometra, plusieurs espèces Helicometra fasciala (Rud., 1819), H. pulchella Rud., 1919), H. gobii (Stossich, 1883) semblent exister dans les Gobiidés, les Labridés, les Anguillidés de l'ẻtang de Thau mais ces espèces ont été placées en synonymie en totalité ou pro-parte par de nombreux auteurs (Palombi, 1929, 1931 ; Nicoll, 1910, Yamaguti, 1971). Nous nous contenterons de nommer ces Helicometra : Helicometra sp. De même Acanthocephaloides propinquus (Dujardin, 1845) a été recensée chez des Poissons d'une trentaine de familles dont beaucoup sont représentées dans cette lagune. Des transferts expérimentaux de parasites adultes et vivants ont été pratiqués entre Poissons de même espèce puis entre Poissons d'espèces différentes.

Les Poissons " hòtes expérimentaux " sont des Gobiidés de l'espèce Gobius nigyer pêchés dans un étang d'eau saumâtre (étang de Mauguio) distant d'une trentaine de kilomètres de l'étang de Thau. Les dissections préparatoires des lots témoins ont permis de montrer que ces Poissons n'étaient jamais infestés par les Helminthes que nous avons étudiés et qu'ils pouvaient donc être considérés comme "sains ». Les raisons de l'absence de Trématodes et d'Acanthocéphales dans les Gobies de l'étang de Mauguio sont peut-être à rechercher dans les facteurs mésologiques différents entre ces deux étangs : l'étang de Mauguio peu profond est beaucoup plus eurytherme et plus euryhalin que l'étang de Thau où les influences marines sont très nettes.

1. Premier symposium sur la spécificité parasitaire des parasites de Vertébrés, 1957, Université de Neuchâtel, Imp. Attinger, 324 p.

2. Deuxième symposium sur la spécificité parasitaire des parasites de Vertébrés, 1982, Mem. Mus. Nal. Hist. nat., Série A, Zool., 123, 326 p. 
Quatre types d'expériences ont été menées en utilisant des Poissons parasités pêchés dans l'étang de Thau :

A. Dans la première série, nous avons fait ingérer aux Grobies expérimentaux des tubes digestifs de Gobius niger renfermant Acanthocephaloides propinquus et Helicometra sp.

Ces expériences reproduisent expérimentalement le cannibalisme naturel qui est assez répandu chez de nombreuses espèces de Poissons, en particulier chez les Gobiidés (Casabianca et Kiener, 1969).

B. Dans la deuxième série, les tubes digestifs donnés aux G. niger "sains" provenaient d'individus d'une autre espèce de Gobie. Zosterisessor ophiocephalus infestée par deux espèces d'Helminthes identiques, semble-t-il à celles de l'expérience précédente.

C. Dans la troisième série, les tubes digestifs appartenaient aux individus d'une espèce de Labridés Symphodus cinereus. Ils étaient porteurs de Helicometra sp. et d'une autre espèce de Trématodes : Peracreadium perezzi, Mathias, 1926.

Ces deux dernières séries pratiquées entre espèces différentes peuvent être assimilées à de l'ichtyophagie interspécifique.

D. Enfin, ces expériences préliminaires ne permettant qu'une étude qualitative, nous les avons répétées en faisant absorber aux Gobies " sains " des gélules en gélatine. Cette technique nous permettant de faire ingérer un nombre connu d'Helminthes aux hôtes expérimentaux nous paraît prometteuse pour des expériences quantitatives ultérieures et il nous a paru indispensable de la tester tout d'abord sur le plan qualitatif.

\section{Résultats}

Les deux premières séries d'expériences ont duré $1,8,14$ et 42 jours et se sont révélés positives. Les individus d'Acanthocephaloides propinquus et d'Helicometra sp. qui parasitaient simultanément les tubes digestifs des hôtes Gobius niger et Zosterisessor ophiocephalus sont passés sans altérations dans les Gobius niger expérimentaux.

La troisième série d'expériences faisait intervenir uniquement des Trématodes parasites du Labridé Symphodus cinereus : Helicometra sp. et Peracreadium perezzi.

Au bout de 5 jours tous les $P$. perezzi avaient disparu et les Helicomelra sp. bien que présents, étaient remplis de globules lipidiques à la place des organes génitaux.

Nous avons obtenu les mêmes résultats positifs avec l'utilisation des gélules.

\section{Discussion}

Les expériences de cannibalisme que nous venons de décrire ont été envisagées à partir de l'observation d'un Gobius niger en voie de digestion dans l'estomac d'un autre Gobius niger pêché dans l'étang de Thau. 
Cette observation et les expériences qui en découlent, nous prouvent que le cannibalisme doit être pris en considération lors d'une étude complète de la dynamique des populations de ces Helminthes intestinaux car de nombreuses espèces de Poissons sont ichtyophages et même cannibales. L'hòte définitif peut être considéré, en effet, comme hôte concentrant les effectifs des parasites. La possibilité chez les poissons d'un transfert d'Acanthocéphales entériques par cannibalisme a déjà été abordé par plusieurs auteurs (Nickol, 1985).

Ce transfert, sans altérations de parasites intestinaux adultes d'un Poisson à l'autre par cannibalisme et par prédation ouvre un champ important d'investigations tant sur le plan qualitatif que sur le plan quantitatif. En effet, il n'est plus nécessaire de connaître le cycle évolutif des parasites étudiés pour tester les problèmes de la spéciation des Helminthes et de leur spécificité vis-à-vis de l'hôte définitif.

Ainsi ces expériences nous permettent de supposer fortement que les Helicometra présents chez les Gobies (G. niger et Z. ophiocephalus) n'appartiennent pas à la même espèce que les Helicometra présents chez le Labre Symphodus cinereus.

Le succès des expériences pratiquées au moyen de gélules de gélatine permet d'envisager l'infestation de Poissons " sains " avec un nombre connu de parasites adultes ou juvéniles, provenant de Poissons de la même espèce ou d'espèce différente, ou bien d'étudier à partir de ces techniques expérimentales le temps de maturation des individus, leur longévité, les compétitions inter- ou intra-spécifiques.

\section{RÉFÉRENCES}

Ayala F. I. : Genetic differenciation during the speciation process. Evolution Biol., 1975, 8, 1-75. Buron I. DE, RENAUd F., Euzet L. : Speciation and specificity of acanthocephalans. Genetic and morphological studies of Acanthocephaloides geneticus sp. nov. parasitizing Arnoglossus laterna (Bothidae) from the Mediterranean littoral (Sète, France). Parasitology, 1986, 92, 165-171.

Casablanca de M. L., Kiener A. : Gobiidae des étangs corses, systématique, écologie, régime alimentaire et position dans les chaines trophiques. Vie Milieu, Série A, Biologie marine, $1969,20(3 / 4), 611-633$.

Palombi A. : Le specie del genere Helicometra essistenti nella collezione elmintologica centrale italiana. Ann. Mus. Zool. R. Univ. Napoli, n. s., 5, 1929, 1-19.

Palombi A. : Il polimorfismo nei trematodi. Ricerche sperimentali su Helicometra fasciata (Rud.) $=$ H. pulchella (Rud.) $=$ H. sinuata (Rud.). Ann. Mus. Zool. R. Univ., n. s., 1931, 6, 1-8.

Pasteur G., Pasteur N. : Les critères biochimiques de l'espèce animale. Les problèmes de l'espèce dans le règne animal. Mem. Soc. Zool. France, 1980, 40, 99-150.

Nickol B. B. : Epizootiology in Biology of the Acanthocephala (Crompton D. W. T., Nickol B. B., Eds.). Cambridge University Press, 1985, 341-346.

Nicoll W. : On the entozoa of fishes from the Firth of Clyde. Parasitology, 1910, 3, 322-359.

Yamaguti $S$. : Synopsis of digenetic trematodes of vertebrates (2 parts). Keigaku publishing Co., Tokyo, 1971, 1, $1074 \mathrm{p}$. 